\title{
In vivo effect of mutations in the antiterminator LacT in Lactobacillus casei
}

\author{
María J. Gosalbes, Carlos D. Esteban and Gaspar Pérez-Martínez
}

Departamento de Biotecnología, Instituto de Agroquímica y Tecnología de Alimentos (CSIC) Polígono de la Coma s/n, Apartado de correos (PO Box) 73, 46100-Burjassot, Valencia, Spain
Author for correspondence: Gaspar Pérez-Martínez. Tel: +34 96 3900022. Fax : +34 963636301. e-mail: gaspar.perez@iata.csic.es

The antiterminator LacT regulates the expression of the lactose operon in Lactobacillus casei and its activity is controlled by EIILac and common PTS elements. LacT shows the two conserved domains (PRD-I and PRD-II) characteristic of the BgIG antiterminator family that are implicated in the regulation of their activity, possibly by phosphorylation of conserved histidines. By site-directed mutagenesis of LacT, four histidines (His-101, His-159 in PRD-I and His-210, His-273 in PRD-II) were replaced by alanine or aspartate, mimicking non-phosphorylated and phosphorylated forms, respectively. These constructions were used to complement $\Delta / a c T$ and $\Delta$ ccpA mutants. L. casei strains ( $\Delta /$ /acT) carrying the replacement of His-101 or His-159 by Ala showed phospho- $\beta$-galactosidase activity in absence of the inducer (lactose), indicating that these amino acids, located in PRD-I, are essential for Ell-dependent induction of the lac operon, possibly by dephosphorylation. Interestingly, these mutations rendered LacT thermosensitive. Moreover, expression of H210A and H273A (PRD-II) mutations in L. casei $\triangle$ ccpA showed that these two histidyl residues could have a role in LacT-dependent carbon catabolite repression (CCR) of this system. Overexpression of LacT in a ccpA background rendered the lac operon insensitive to CCR, but it was still sensitive to lactose induction. This suggests that the transfer of phosphate groups from PTS elements, which controls these two regulatory processes (CCR and substrate induction), could have different affinity for PRD-I and PRD-II histidines.

Keywords: L. casei, lactose induction, carbon catabolite repression, mutagenesis, phosphorylation

\section{INTRODUCTION}

In lactic acid bacteria, lactose transport and metabolism can be mediated by several pathways. A lactose-specific phosphoenol-pyruvate-dependent phosphotransferase system (PTS) and phospho- $\beta$-galactosidase (P- $\beta$-gal) have been described in the genera Staphylococcus, Streptococcus, Lactococcus and Lactobacillus (de Vos \& Vaughan, 1994). In Lactobacillus casei, lactose genes are transcribed as an operon, lacTEGF, encoding an antiterminator (LacT), lactose-specific PTS proteins (LacE and LacF) and a P- $\beta$-gal (LacG) (Alpert \& Chassy, 1988, 1990; Porter \& Chassy, 1988; Alpert \& Siebers,

Abbreviations: CCR, carbon catabolite repression; $\mathrm{P}-\beta$-gal, phospho- $\beta$ galactosidase; PRD, PTS regulation domain; PTS, phosphoenol-pyruvatedependent phosphotransferase system; RAT, ribonucleic antiterminator.
1997; Gosalbes et al., 1997). In this micro-organism, the lac operon is subject to induction by lactose through a mechanism of transcriptional antitermination in which LacT activity is negatively regulated by the lactosespecific enzymes, EII ${ }^{\mathrm{Lac}}$ (Gosalbes et al., 1999). Moreover, the expression of this operon is repressed by glucose and other rapidly metabolizable carbon sources, partly through CcpA-mediated carbon catabolite repression (CCR) (Monedero et al., 1997; Viana et al., 2000). However, it has been shown that LacT and PTS elements are involved in an additional CcpA-independent CCR mechanism (Gosalbes et al., 1999).

Antitermination activity has been extensively studied in homologous proteins, such as BglG of Escherichia coli (Mahadevan \& Wright, 1987; Mahadevan et al., 1987; Schnetz et al., 1987; Schnetz \& Rak, 1988, 1990; Amster-Choder et al., 1989; Houman et al., 1990; 
Amster-Choder \& Wright, 1993; Görke \& Rak, 1999) and SacT, SacY, LicT and GlcT of Bacillus subtilis (Zukowski et al., 1990; Crutz et al., 1990; Débarbouillé et al., 1990; Arnaud et al., 1992, 1996; Crutz \& Steinmetz, 1992; Le Coq et al., 1995; Krüger \& Hecker, 1995; Schnetz et al., 1996; Rutberg, 1997; Stülke et al., 1997; Tortosa et al., 1997; Bachem \& Stülke, 1998; Idelson \& Amster-Choder, 1998). In lactic acid bacteria, only two other antiterminator proteins have been reported: BglR in Lactococcus lactis and, recently, BglG in Lactobacillus plantarum (Bardowski et al., 1994; Marasco et al., 2000). Antitermination activity requires binding of these proteins to a ribonucleic antiterminator (RAT) sequence in the mRNA, stabilizing a stem-loop structure that prevents the formation of the transcriptional terminator located in the leader fragment preceding the coding regions (Aymerich \& Steinmetz, 1992). The antiterminators show a modular organization, with an RNA-binding region and two conserved PTS regulation domains (PRD-I and PRD-II), which, as demonstrated for BglG, LicT, SacT and SacY, contain phosphorylatable histidyl residues (two in each domain) that render them susceptible to regulation by PTS elements (Stülke et al., 1998; Tortosa et al., 1997). It has been proposed that PRD-I is negatively regulated by the sugar-specific PTS elements, EII, and PRD-II could be subject to a positive control by the general PTS protein, $\mathrm{HPr}$ (Stülke et al., 1998). Biochemical and genetic studies allowed the phosphorylation sites in different regulators to be identified. However, despite the fact that they are well conserved, they have different regulatory roles. The antiterminators SacT, GlcT and LicT from Bacillus subtilis are negatively controlled by EII-dependent phosphorylation of one histidyl residue in PRD-I (His97, His-104 and His-100, respectively) (Arnaud et al., 1992, 1996; Le Coq et al., 1995; Bachem \& Stülke, 1998). However, in the PRD-containing activators LicR and LevR of B. subtilis, and MtlR of Bacillus stearothermophilus, the phosphorylation sites involved in this regulatory mechanism are located in the EIIA-like domain and PRD-II (Martin-Verstraete et al., 1998; Tobisch et al., 1999; Henstra et al., 2000). In addition, some of these regulators (SacT, LicT and LevR) are activated by HPr-dependent phosphorylation at different sites (Martin-Verstraete et al., 1995, 1998; Arnaud et al., 1996; Lindner et al., 1999). In E. coli, the antiterminator $\mathrm{BglG}$ regulates the expression of the $b g l$ operon involved in $\beta$-glucoside transport. EII ${ }^{\mathrm{Bgl}}$ transports and phosphorylates $\beta$-glucosides but it also modulates BglG activity by phosphorylation. The His-208 of this antiterminator, located in PRD-II, was identified as the phosphorylation site (Chen et al., 1997, 2000). On the other hand, it has been recently been shown that HPr also phosphorylates BglG, but the presumptive sites remain unidentified (Görke \& Rak, 1999).

The aim of this work was to investigate the role of the conserved histidyl residues (His-101, His-159, His-210 and His-273) of LacT in the mechanisms that control antiterminator activity in Lactobacillus casei. For this purpose, the potential phosphorylation sites of LacT were replaced by alanine or aspartate by site-directed mutagenesis. The effect of these mutations on the expression of the lac operon was evaluated by determining $\mathrm{P}-\beta$-gal activity from cultures grown under different induction/repression conditions.

\section{METHODS}

Plasmids, bacterial strains and growth conditions. Lactobacillus casei strains and plasmids used in this work are listed in Table 1. L. casei cells were grown in MRS medium (Oxoid) and MRS fermentation broth (Adsa-Micro) plus $0.5 \%(\mathrm{w} / \mathrm{v}$ ) of the different carbohydrates at $37^{\circ} \mathrm{C}$ and $30^{\circ} \mathrm{C}$ under static conditions. E. coli $\mathrm{DH} 5 \alpha$ was grown with shaking at $37^{\circ} \mathrm{C}$ in Luria-Bertani (LB) medium. Plating of bacteria was performed on the respective media solidified with $1.5 \%$ agar. When required, the concentrations of antibiotics used were $100 \mu \mathrm{g}$ ampicillin and $300 \mu \mathrm{g}$ erythromycin $\mathrm{ml}^{-1}$ to select E. coli transformants and $5 \mu \mathrm{g}$ erythromycin $\mathrm{ml}^{-1}$ for L. casei.

Recombinant DNA procedures. Genomic DNA from L. case $i$ was purified using a Purogene DNA Isolation Kit (Gentra Systems), following the procedure described by the manufacturer. Restriction and modifying enzymes were purchased commercially and used according to the recommendations of the manufacturers. General cloning procedures were performed as described by Sambrook et al. (1989). L. casei strains were transformed by electroporation with a Gene-pulser apparatus (Bio-Rad) as described elsewhere (Posno et al., 1991). PCR was performed using the Expand High-Fidelity PCR system (Roche Molecular Biochemicals), containing $200 \mu \mathrm{M}$ of each deoxynucleoside triphosphate and $10 \mathrm{pmol}$ of each primer. After agarose gel electrophoresis, the amplified DNA was recovered with a GFX PCR Kit (Amersham Pharmacia Biotech).

Construction of a $\Delta$ lact mutant. Deletion of lacT was obtained by Campbell-like recombination using the integrative plasmid pRV300 (Leloup et al., 1997). Two fragments of 500 and $543 \mathrm{bp}$ spanning the regions upstream and downstream of lacT, respectively, were obtained by PCR using total DNA of L. casei as template. The primers used were lac50 (5'-GAGCGGTACCATCAGTAGA-3') and lac51 (5'-GTTGTCATCCCCTCCCAG-3') for one fragment and lac52 (5'-CGCGCTCGCAGTCGTAGA-3') and lac25 (5'CGATATGAGCTCAGATC-3') for the other fragment. The two PCR products were ligated and the ligation mix used as template in a PCR with lac50 and lac25. These two oligonucleotides carried $K p n \mathrm{I}$ and $\mathrm{SacI}$ sites (underlined), respectively, allowing the cloning of the last PCR product into $K p n \mathrm{I} /$ Sacl-digested pRV300, giving pRV $\Delta$ lacT. This plasmid was used to transform L. casei. A $\mathrm{Lac}^{+}$and $\mathrm{Er}^{+}$transformant was grown for 200 generations without antibiotic in order to allow the second recombination event, which would excise the vector, giving $\mathrm{Lac}^{-} \mathrm{Er}^{-}$colonies. The correct first and second recombination events were confirmed by PCR. One of the lacT mutants was selected and the strain named BL195. In this strain the lacT gene was totally deleted, while keeping the lac promoter, including all the initiation signals and regulatory sites unaltered upstream of the remaining genes of the lac operon.

Plasmid constructions and site-directed mutagenesis. To obtain the plasmid pLacT, lacT was amplified with primers lac53 (5'-GCACTGGGAGGGGATGACAA-3') and lac54 (5'TTGGAAGCTTGCTTCAAAGCC-3') using L. casei DNA as template, and the PCR product was cloned into the SmaI site of pUC18 using a Sureclone kit (Amersham Pharmacia Biotech). The lac54 primer had two substitutions (boldface) to 
Table 1. L. casei strains and plasmids used in this study

\begin{tabular}{|c|c|c|}
\hline Strain or plasmid & Description & Source or reference \\
\hline \multicolumn{3}{|l|}{ L. casei } \\
\hline CECT $5275^{*}$ & Wild-type & B. Chassy \\
\hline PL20 & CECT 5275(pGAL9) & This work \\
\hline BL195 & $\Delta l a c T$ & This work \\
\hline BL190 & $\Delta c c p A$ & C. D. Esteban \\
\hline \multicolumn{3}{|l|}{ Plasmids } \\
\hline pRV300 & $\operatorname{Er}^{\mathrm{r}}$ & Leloup et al. (1997) \\
\hline $\mathrm{pRV} \Delta \mathrm{lacT}$ & $\begin{array}{l}\text { pRV300 with two fragments spanning the regions } \\
\text { downstream and upstream of lacT }\end{array}$ & This work \\
\hline pUC18 & $\mathrm{Am}^{\mathrm{r}}$ & $\begin{array}{l}\text { Amersham Pharmacia } \\
\text { Biotech }\end{array}$ \\
\hline pLacT & pUC18 with $\operatorname{lac} T$ & This work \\
\hline pLacTH101A & pLacT derivative (codon 101 of lacT is GCT for Ala) & This work \\
\hline pLacTH101D & pLacT derivative (codon 101 of $\operatorname{lacT}$ is GAT for Asp) & This work \\
\hline pLacTH159A & pLacT derivative (codon 159 of $\operatorname{lacT}$ is GCC for Ala) & This work \\
\hline pLacTH210A & pLacT derivative (codon 210 of $\operatorname{lacT}$ is GCT for Ala) & This work \\
\hline pLacTH210D & pLacT derivative (codon 210 of lacT is GAT for Asp) & This work \\
\hline pLacTH273A & pLacT derivative (codon 273 of $\operatorname{lacT}$ is GCC for Ala) & This work \\
\hline pGAL9 & $\operatorname{Er}^{r}$ & $\begin{array}{l}\text { Pérez-Martínez et al. } \\
\text { (1992) }\end{array}$ \\
\hline pGALT & pGAL9 derivative expressing LacT & This work \\
\hline pGALTH101A & pGAL9 derivative expressing LacTH101A & This work \\
\hline pGALTH101D & pGAL9 derivative expressing LacTH101D & This work \\
\hline pGALTH159A & pGAL9 derivative expressing LacTH159A & This work \\
\hline pGALTH210A & pGAL9 derivative expressing LacTH210A & This work \\
\hline pGALTH210D & pGAL9 derivative expressing LacTH210D & This work \\
\hline pGALTH273A & pGAL9 derivative expressing LacTH273A & This work \\
\hline
\end{tabular}

*CECT, Colección Española de Cultivos Tipo.

First PCR

Second PCR

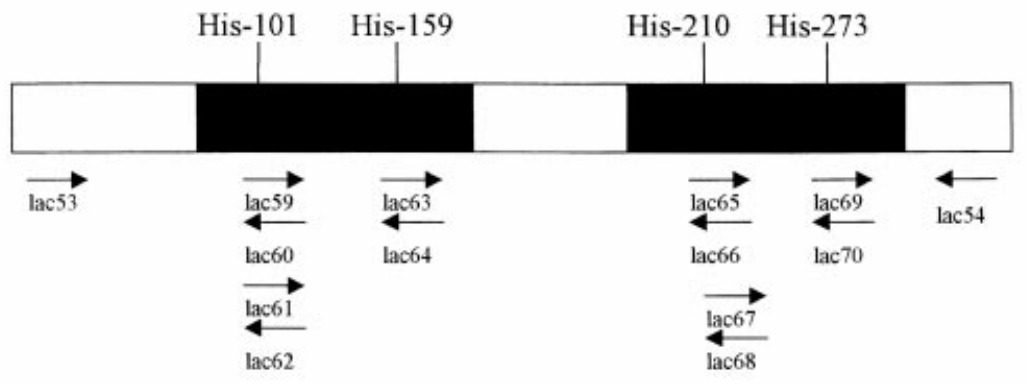

mutations

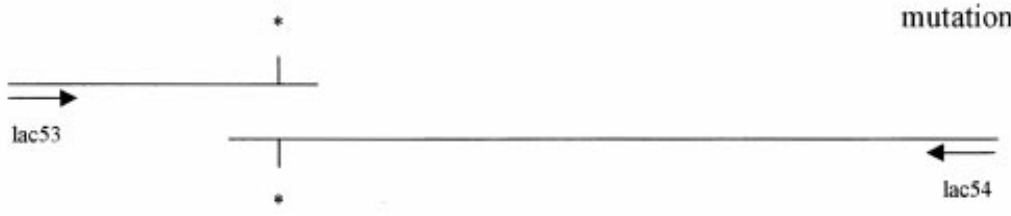

(H101A (lac59,lac60) H101D (lac61,lac62) H159A (lac63,lac64) H210A (lac65,lac66) H210D (lac67,lac68) H273A (lac69, lac70)

Fig. 1. Site-directed mutagenesis and strategy of plasmid construction. The bar represents the LacT protein, with the two PRDs (in black) and four putative phosphorylation sites, His-101, His-159, His-210 and His-273. Arrows indicate oligonucleotides that were used in the PCRs as primers. A point mutation $(*)$ is indicated as example. The resulting PCR fragments containing the mutations are indicated as H101A (His-101 replaced by alanine), H101D (His-101 replaced by aspartate), H159A (His-159 replaced by alanine), H210A (His-210 replaced by alanine), H210D (His-210 replaced by aspartate) and H273A (His-273 replaced by alanine). These fragments were cloned into pUC18 and subcloned into pGAL9 vector, giving pGALT, pGALTH101A, pGALTH101D, pGALTH159A, pGALTH210A, pGALTH210D and pGALTH273A, which contained wild-type lacT and the mutated genes, respectively (see Table 1 ). These plasmids were used to transform $L$. casei CECT5275, the $\Delta$ lacT strain (BL195) and the ccpA mutant (BL190). 
introduce a new HindIII site (underlined). pLacT was used as template in the site-directed mutagenesis to replace the triplets encoding histidyl residues of LacT by alanine or aspartate (H101A, H101D, H159A, H210A, H210D and H273A). These amino acids (Ala and Asp) mimic non-phosphorylated and phosphorylated forms of the residue. The overlap extension method applied allows introduction of site-specific mutations and generation of a mutant gene in two PCR steps (Ho et al., 1989). In the first PCR step, two fragments of lacT were independently amplified using outside primers (lac53 or lac54) and the appropiate oligonucleotide of the following mutagenic primers (lac59 5'-TGAGTGATGCTATTTACGA and lac60 5'-TCGTAAATAGCATCACTCA, lac61 5'-TGAGTGATGATATTTACGA and lac62 5'-TCGTAAATATCATCACTCA, lac63 5'-TGGCGTTGGCCTTTATCAA and lac64 5' TTGATAAAGGCCAACGCCA, lac65 5'-TTATGATTGCTCTCCAGTA and lac66 5'-TACTGGAGAGCAATCATAA, lac67 5'-TTATGATTGATCTCCAGTA and lac68 5'TACTGGAGATCAATCATAA, lac69 5'-TCATCATCGCCATTCAGCG and lac70 5'-CGCTGAATGGCGATGATGA) (Fig. 1). The mutated triplets are in boldface. These pairs of primers are complementary, hence the strands of the fragments obtained overlapped and they could be extended in a subsequent reaction using lac53 and lac54 primers. The products of these second PCRs were cloned into pUC18/SmaI. The resulting plasmids were named pLacH101A, pLacH101D, pLacH159A, pLacH210A and pLacH210D (Fig. 1). To replace His-273 by an Ala residue, lac2 (5'-CAACGATATAAGCGCAGATC) was used as external primer. The strategy was followed as described above and the plasmid obtained was designated pLacH273A (Fig. 1). The orientation of the inserts in pUC18 vector was checked by digestion with the endonuclease HindIII. The fragments were subsequently sequenced to exclude PCR artifacts using a Perkin-Elmer Abi Prism 310 automated sequencer. Plasmids carrying wild-type and mutated genes were digested with BamHI and HindIII and the resulting fragment cloned into BamHI/HindIIIdigested pGAL9 (Pérez-Martínez et al., 1992), generating pGALT, pGALTH101A, pGALTH101D, pGALTH159A, pGALTH210A, pGALTH210D and pGALTH273A. In these pGAL9-derived plasmids lacT variants are under control of the constitutive SPO2/AL9 promoter. These plasmids were used to transform L. casei CECT5275, the $\Delta$ lacT strain (BL195) and the ccpA mutant (C. D. Esteban, unpublished data) (Fig. 1). The L. casei CECT5275(pGAL9) transformant was named PL20.

P- $\boldsymbol{\beta}$-gal assay. Activity was assayed as previously described (Chassy \& Thompson, 1983). L. casei cells were permeabilized by vortexing for $5 \mathrm{~min}$ with $10 \mathrm{mM}$ deoxycholic acid (Taranto, 1999).

\section{RESULTS}

\section{Effect of mutations in His-101 and His-159 on LacT activity}

LacT activity is negatively regulated by $\mathrm{EII}^{\mathrm{Lac}}$, as $L$. casei mutants in these elements displayed an inducerindependent antiterminator activity (Gosalbes et al., 1999). The conserved histidyl residues of PRD-I were replaced by Ala (H101A and $\mathrm{H} 159 \mathrm{~A}$ ) and by Asp (H101D) to study their role in the induction mechanism of the lac operon. These lacT mutant genes were cloned under a constitutive promoter SPO2/AL9 in pGAL9 (Table 1, Fig. 1) and were used to complement a mutant with a chromosomal deletion of lacT (BL195), obtained by Campbell-like recombination. This $\Delta l a c T$ mutant could not be grown on lactose, since it lacked the antiterminator. BL195 was transformed with pGALT, pGALTH101A, pGALTH101D and pGALTH159A, and its complementation was initially tested on MRS fermentation plates with lactose. While BL195(pGALT), expressing wild-type lacT from the SPO2/AL9 promoter, and the wild-type strain (PL20) fermented this sugar, the same strain transformed with plasmids bearing mutated lacT [BL195(pGALTH101A), BL195(pGALTH101D) and BL195(pGALTH159A)] did not utilize lactose at $37^{\circ} \mathrm{C}$. However, surprisingly, when grown at $30^{\circ} \mathrm{C}$ BL195(pGALTH101A) and BL195(pGALTH159A) showed a $\mathrm{Lac}^{+}$phenotype. In order to confirm this finding, $\Delta l a c T$ transformants carrying one of these two mutations (H101A or H159A) were grown at $37^{\circ} \mathrm{C}$ and $30^{\circ} \mathrm{C}$ on MRS fermentation broth supplemented with lactose and the P- $\beta$-gal activity was determined, as a measure of the lac operon expression. Neither BL195(pGALTH101A) nor BL195(pGALTH159A) showed activity when grown at $37^{\circ} \mathrm{C}\left[4.66 \pm 0.30\right.$ and $1 \cdot 18 \pm 0.64 \mathrm{nmol} \mathrm{min}{ }^{-1}$ (mg dry weight $)^{-1}$, respectively], but a significant activity was detected at $30{ }^{\circ} \mathrm{C}[22 \cdot 55 \pm 3 \cdot 37$ and $23 \cdot 45 \pm 5 \cdot 48 \mathrm{nmol}$ $\min ^{-1}$ (mg dry weight $)^{-1}$, respectively].

The involvement of His-101 and His-159 in the induction mechanism of the lac operon was studied in vivo by determining P- $\beta$-gal activity in BL195(pGALT), BL195(pGALTH101A), BL195(pGALTH101D), BL195(pGALTH159A) and wild-type strains, when grown on ribose (non-inducing sugar), lactose (inducer) and glucose plus lactose (CCR) (Table 2). In BL195(pGALT), the expression of lac operon was restored in lactose-grown cells, although P- $\beta$-gal activity was lower than that found in the wild-type strain (PL20). Both strains, BL195(pGALT) and PL20, showed the same pattern of P- $\beta$-gal expression: induction by lactose and repression by glucose. This suggested that PRD-I from LacT could be dephosphorylated by EII ${ }^{\mathrm{Lac}}$ even when it is overexpressed. In the absence of lactose (ribose-grown cells), transformants with Ala replacements [BL195(pGALTH101A) and BL195(pGALTH159A)] showed about half the activity detected on lactose, suggesting an additive effect of these mutations. Alternatively, if each of the Ala replacements in PRD-I could render a fully active LacT, the structural instability provoked by these mutations may be the cause for the reduced activity detected. In contrast, no, or negligible, activity was detected in BL195(pGALTH101D) grown on any of the three sugars used (Table 2), indicating that the phosphorylation of this residue may inactivate LacT, which would complement the results obtained with the Ala replacements.

\section{Characterization of putative phosphorylation sites in the PRD-II of LacT}

Sequence alignment of proteins belonging to the BglG family indicated His-210 and His-273 of LacT as potential phosphorylation sites. Thus, strains 
Table 2. P- $\beta$-gal activity of different strains

\begin{tabular}{|lccc|}
\hline Strain & \multicolumn{3}{c|}{ P- $\boldsymbol{\beta}$-gal activity* of cells grown on: } \\
\cline { 2 - 4 } & Lactose & Glucose + lactose & Ribose \\
\hline PL20 & $36 \pm 2 \cdot 56$ & ND & $0 \cdot 84 \pm 0 \cdot 43$ \\
BL195(pGAL9) & ND & ND & $1 \cdot 29 \pm 0 \cdot 15$ \\
BL195(pGALT) & $19 \cdot 34 \pm 5 \cdot 86$ & $2 \cdot 02 \pm 0 \cdot 73$ & $1 \cdot 88 \pm 0 \cdot 25$ \\
BL195(pGALTH101A)† & $22 \cdot 55 \pm 3 \cdot 37$ & $1 \cdot 20 \pm 0 \cdot 44$ & $13 \cdot 07 \pm 4 \cdot 02$ \\
BL195(pGALTH101D) & ND & $1 \cdot 40 \pm 0 \cdot 66$ & $1 \cdot 34 \pm 0 \cdot 60$ \\
BL195(pGALTH159A)† & $23 \cdot 45 \pm 5 \cdot 48$ & $1 \cdot 94 \pm 0 \cdot 45$ & $12 \cdot 35 \pm 3 \cdot 70$ \\
BL195(pGALTH210A) & ND & $2 \cdot 3 \pm 0 \cdot 34$ & $2 \cdot 15 \pm 1 \cdot 06$ \\
BL195(pGALTH210D) & ND & $1 \cdot 66 \pm 0 \cdot 70$ & $0 \cdot 74 \pm 0 \cdot 22$ \\
BL195(pGALTH273A) & ND & $0 \cdot 60 \pm 0 \cdot 13$ & $0 \cdot 97 \pm 0 \cdot 32$ \\
BL190(pGAL9) & $44 \cdot 60 \pm 3 \cdot 65$ & ND & ND \\
BL190(pGALT) & $44 \cdot 86 \pm 3 \cdot 22$ & $40 \cdot 90 \pm 0 \cdot 58$ & ND \\
BL190(pGALTH210A) & $49 \cdot 49 \pm 2 \cdot 5$ & ND & ND \\
BL190(pGALTH210D) & $46 \cdot 63 \pm 1 \cdot 45$ & $16 \cdot 26 \pm 0 \cdot 96$ & ND \\
BL190(pGALTH273A) & $48 \cdot 42 \pm 3 \cdot 25$ & ND & ND \\
\hline
\end{tabular}

* $\mathrm{P}$ - $\beta$-gal activity is expressed as nmol $o$-nitrophenyl $\beta$-D-galactopyranoside 6-phosphate hydrolysed $\min ^{-1}(\mathrm{mg} \text { dry weight })^{-1}$. The values and standard deviations are from at least three independent experiments. ND, Not detected.

†P- $\beta$-gal activity from BL195(pGALTH101A) and BL195(pGALTH159A) corresponds to the cultures grown at $30^{\circ} \mathrm{C}$ (all other values are from cultures grown at $37^{\circ} \mathrm{C}$ ).

[BL195(pGALTH210A), BL195(pGALTH210D) and BL195(pGALTH273A)] were constructed with the His-210 and His-273 residues replaced by Ala or Asp, mimicking non-phosphorylated and phosphorylated forms, respectively. In this case, thermosensitivity was also tested on MRS fermentation plates supplemented with lactose at $37^{\circ} \mathrm{C}$ and $30^{\circ} \mathrm{C}$. None of the mutants grew on lactose at either temperature. In fact, no, or negligible, P- $\beta$-gal activity was detected on ribose- or glucose-plus-lactose-grown cells in these mutants (Table 2).

To study the involvement of PRD-II (His-210 and His273) in CCR, a $\triangle c c p A$ mutant (BL190) was transformed with pGAL9, pGALT, pGALTH210A, pGALTH210D and pGALTH273A. All the transformants showed P- $\beta$ gal activity in lactose-grown cells due to the normal expression of the chromosomal lac operon in the host strain BL190. However, any effect on the expression of the lac operon in BL190 on glucose-plus-lactose medium would be exclusively due to LacT-mediated CCR. Expression of LacT from a multicopy plasmid (pGALT) in the $c c p A$ mutant conferred glucose insensitivity, possibly due to dephosphorylation of PRD-II when the inducer was present and in conditions of LacT molar excess. Expression of the mutation H210D in BL190 resulted in the activation of the operon in the presence of glucose and the inducer, lactose, indicating that this mutation was partially active and insensitive to glucose repression in the $c c p A$ mutant. However, Ala replacements did not give an active form of antiterminator protein, since the repressing effect of glucose on
P- $\beta$-gal activity remained in BL190(pGALH210A) and BL190(pGALH273A) (Table 2).

\section{DISCUSSION}

The antiterminator LacT regulates lactose operon (lacTEGF) expression in L. casei. The activity of this protein is controlled by EII ${ }^{\mathrm{Lac}}$ and PTS elements in response to the availability of lactose or the presence of glucose (Gosalbes et al., 1999; Monedero et al., 1997; Viana et al., 2000). Sequence analysis of the members of the BglG family revealed a modular organization, with an RNA-binding domain and PTSregulated domains (PRD-I and PRD-II) with usually four well-conserved potential phosphorylation sites (two in each domain). PRD-containing regulators could be subject to a dual regulation by phosphorylation: negative control by EII exerted on PRD-I and positive control by HPr-His-P generally on PRD-II. Therefore, His-101, His-159 in PRD-I and His-210, His-273 in PRD-II of LacT were mutated to investigate their role in the phosphorylation-dependent regulatory mechanisms.

BL195 ( $\Delta l a c T)$ had a complete deletion of lacT, and therefore it had a $\mathrm{Lac}^{-}$phenotype. The operator, RAT sequence and terminator structure remained functional, as demonstrated by the fact that constitutive expression of LacT from pGALT partially restored growth on lactose and gave the same expression pattern as the wild-type (glucose repression and induction by lactose). However, in other works reporting overproduction of antiterminators, such as LicT, substrate induction was 
eliminated (Krüger \& Hecker, 1995; Krüger et al., 1996).

Antiterminator activity of all members of the BglG family of antiterminators has been shown to be subject to substrate induction through phosphate exchange with the sugar-specific EII PTS elements (Stülke et al., 1998). In L. casei, transcriptional studies of the lac operon in lacE and lacF mutants showed an inducer-independent antiterminator activity, indicating a negative control of these elements (Gosalbes et al., 1999). According to the model of PTS-mediated regulation of antiterminators (Stülke et al., 1998), EII ${ }^{\mathrm{Lac}}$ would dephosphorylate LacT PRD-I in the presence of lactose to yield its active form. EII-dependent negative control has been demonstrated to involve at least one of two conserved histidines of PRD-I in LicT, SacY, SacT and GlcT (His-101, His-99, His-97 and His-104, respectively) (Schnetz et al., 1987; Débarbouillé et al., 1990; Arnaud et al., 1992, 1996; Stülke et al., 1997; Bachem \& Stülke, 1998). PRDI alignment of different antiterminators located highly conserved histidines at positions His-101 and His-159 in LacT. In this work, the replacement of His-101 or His159 by Ala rendered LacT active in the absence of lactose (ribose-grown cells), although the P- $\beta$-gal activity observed in these strains [BL195(pGALTH101A) and BL195(pGALTH159A)] was lower than that detected on lactose-grown cells. It could be proposed that both residues were involved in the lactose-induction process and one or both of them should be dephosphorylated to obtain full LacT activity. These replacements of His-101 and His-159 by Ala yielded the same phenotype of thermosensitive LacT, suggesting that these histidyl residues may interact and be playing a role in the conformational stability of PRD-I in LacT, as has recently been proposed from the resolved structure of PRDs in LicT (van Tilbeurgh et al., 2001).

LacT activity has also been shown to be sensitive to the presence of glucose, which caused the already reported CcpA-independent CCR of the L. casei lac operon (Monedero et al., 1997; Gosalbes et al., 1999; Viana et al., 2000). In homologous antiterminators, this event apparently required phosphorylation of PRD-II by HPrHis-P, although this effect varied slightly among them. Whereas SacT and LicT are subject to a positive control by HPr-dependent phosphorylation, SacY and GlcT activities do not require it. LicT can be phosphorylated by $\mathrm{HPr}$ at three sites, one in PRD-I (His-159) and the other two in PRD-II (His-207 and His-269). Furthermore, His-207 is preferentially phosphorylated, indicating a fine modulation of LicT activity (Lindner et al., 1999). In contrast, HPr-dependent phosphorylation at the equivalent sites (His-207 and His-269) in SacY does not affect its activity (Tortosa et al., 1997). The total loss of activity in BL195(pGALTH210A) and BL195(pGALTH273A) when grown on lactose suggested that both His residues (His-210 and His-273) should be phosphorylated to obtain the active form of LacT. All the mutants and the wild-type showed a remarkable glucose repression effect, as expression of the lac operon is also repressed by CcpA-dependent
CCR in this genetic background ( $\triangle$ lacT). Thus, we studied the involvement of His-210 and His-273 in LacT-mediated CCR using a $c c p A$ mutant. Strikingly, a derepressed phenotype was observed in BL190(pGALT), possibly due to the overexpression of LacT from a constitutive promoter in a high-copy-number plasmid. Also, a likely expression of the lac operon was found when the mutant protein $\mathrm{H} 210 \mathrm{D}$ was expressed in a $c c p$ A background, although the activity was lower than expected if the operon in this mutant was fully activated or insensitive to the glucose effect, as observed in LicT (van Tilbeurgh et al., 2001). Although this mutation was not temperature sensitive, it may introduce conformational changes or difficulties in dimer formation, which is apparently required for activity in E. coli BglG (Amster-Choder \& Wright, 1992, 1993; Boss et al., 1999). In E. coli it has recently been proposed that HPrdependent phosphorylation of PRD-II is necessary for formation of the dimer (active) form of BglG (Görke \& Rak, 1999), and a similar model was initially suggested for LicT (Krüger \& Hecker, 1995; Krüger et al., 1996). However, recent data obtained from the crystallized regulatory domains of LicT (van Tilbeurgh et al., 2001) showed that phosphorylated histidines in PRDs face inwards, hidden from interactions with the paired molecule in the dimer. Hence, the negative charge introduced in $\operatorname{LacT}(\mathrm{H} 210 \mathrm{D})$ might introduce structural changes that indirectly alter the dimer interface. In BL190(pGALTH210D), the overproduced LacT(H210D) may be forming heterodimers with the chromosomally encoded LacT with weak antiterminator activity. In contrast, in BL195 ( $\operatorname{llacT}$ ) only an unstable homodimer would be formed from pGALTH210D. Nevertheless, the role of the PRD-II histidines in glucose inactivation of LacT has been confirmed with the H210A and H273A mutants as they lack activity in any background.

Further studies are required to clarify the mechanism by which phosphorylation regulates dimerization and activity of LacT. Furthermore, in vitro phosphorylation experiments with the different purified forms of LacT will be necessary to confirm the proposed phosphorylation processes.

In conclusion, we have determined the in vivo effect of site-directed mutations of the LacT antiterminator on lac operon expression in L. casei. Dephosphorylated PRD-I histidyl residues, His-101 and His-159, are indispensable for full induction of lac operon, whilst His-210 and His-273 of PRD-II play a role in CcpAindependent carbon catabolite repression. The results obtained suggest that there could be differences in the affinity of different phosphotransfer elements for PRD-I and PRD-II histidines.

\section{ACKNOWLEDGEMENTS}

We thank D. Coloma for her technical assistance with the automated sequencer. C.D.E. was the recipient of a fellowhip from the Spanish government. This work was financed by the EU project BIO4-CT96-0380 and by funds of the Spanish 
CICyT (Interministerial Commision for Science and Technology) (ref. ALI 98-0714).

\section{REFERENCES}

Albert, T. (1989). Mutational effects on protein stability. Annu Rev Biochem 58, 765-798.

Alpert, C.-A. \& Chassy, B. M. (1988). Molecular cloning and nucleotide sequence of the factor III ${ }^{\text {lac }}$ gene of Lactobacillus casei. Gene 62, 277-288.

Alpert, C.-A. \& Chassy, B. M. (1990). Molecular cloning and DNA sequence of $l a c E$, the gene encoding the lactose-specific enzyme II of the phosphotransferase system of Lactobacillus casei. J Biol Chem 265, 22561-22568.

Alpert, C.-A. \& Siebers, U. (1997). The lac operon of Lactobacillus casei contains lacT, a gene coding for a protein of the BglG family of transcriptional antiterminators. J Bacteriol 179, 1555-1562.

Amster-Choder, O. \& Wright, A. (1992). Modulation of dimerization of a transcriptional antiterminator protein by phosphorylation. Science 257, 1395-1397.

Amster-Choder, O. \& Wright, A. (1993). Transcriptional regulation of the $b g l$ operon of Escherichia coli involves phosphotransferase system-mediated phosphorylation of a transcriptional antiterminator. J Cell Biochem 51, 83-90.

Amster-Choder, O., Houman, F. \& Wright, A. (1989). Protein phosphorylation regulates transcription of the $\beta$-glucoside utilization operon in E. coli. Cell 58, 847-855.

Arnaud, M., Vary, P., Zagorec, M., Klier, A., Débarbouillé, M., Postma, P. \& Rapoport, G. (1992). Regulation of the sacPA operon of Bacillus subtilis: identification of phosphotransferase system components involved in SacT activity. J Bacteriol 174, 3161-3170.

Arnaud, M., Débarbouillé, M., Rapoport, G., Saier, M. H., Jr \& Reizer, J. (1996). In vitro reconstitution of transcriptional antiterminator by the SacT and SacY proteins of Bacillus subtilis. J Biol Chem 271, 18966-18972.

Aymerich, S. \& Steinmetz, M. (1992). Specificity determinants and structural features in the RNA target of the bacterial antiterminator proteins of the BglG/SacY family. Proc Natl Acad Sci US A 89, 10410-10414.

Bachem, S. \& Stülke, J. (1998). Regulation of the Bacillus subtilis GlcT antiterminator protein by components of the phosphotransferase system. J Bacteriol 180, 5319-5326.

Bardowski, J., Ehrlich, S. D. \& Chopin, A. (1994). BglR protein, which belongs to the BglG family of transcriptional antiterminators, is involved in $\beta$-glucoside utilization in Lactococcus lactis. J Bacteriol 176, 5681-5685.

Boss, A., Nussbaum-Shochat, A. \& Amster-Choder, O. (1999). Characterization of the dimerization domain in BglG, an RNAbinding transcriptional antiterminator from Escherichia coli. J Bacteriol 181, 1755-1766.

Chassy, B. M. \& Thompson, J. (1983). Regulation of lactosephosphoenolpyruvate-dependent-phosphotransferase system and $\beta$-D-phosphogalactosidase galactohydrolase activities in Lactobacillus casei. J Bacteriol 154, 1195-1203.

Chen, Q., Engelberg-Kulka, H. \& Amster-Choder, O. (1997). The localization of the phosphorylation site of $\mathrm{BglG}$, the response regulator of the Escherichia coli bgl sensory system. J Biol Chem 272, 17263-17268.

Chen, Q., Postma, P. W. \& Amster-Choder, O. (2000). Dephosphorylation of Escherichia coli transcriptional antiterminator $\mathrm{BglG}$ by the sugar sensor BglF is the reversal of its phosphorylation. J Bacteriol 182, 2033-2036.
Crutz, A. M. \& Steinmetz, M. (1992). Transcription of the Bacillus subtilis sacX and sacY genes, encoding regulators of sucrose metabolism, is both inducible by sucrose and controlled by the DegS-DegU signalling system. J Bacteriol 174, 6087-6095.

Crutz, A. M., Steinmetz, M., Aymerich, S., Richter, R. \& Le Coq, D. (1990). Induction of levansucrase in Bacillus subtilis: an antitermination mechanism negatively controlled by the phosphotransferase system. J Bacteriol 172, 1043-1050.

Débarbouillé, M., Arnaud, M., Fouet, A., Klier, A. \& Rapoport, G. (1990). The sacT gene regulating the sacPA operon in Bacillus subtilis shares strong homology with transcriptional antiterminators. J Bacteriol 172, 3966-3973.

de Vos, W. M. \& Vaughan, E. E. (1994). Genetics of lactose utilization in lactic acid bacteria. FEMS Microbiol Rev 15, 217-237.

Görke, B. \& Rak, B. (1999). Catabolite control of Escherichia coli regulatory protein BglG activity by antagonistically acting phosphorylations. EMBO J 18, 3370-3379.

Gosalbes, M. J., Monedero, V., Alpert, C.-A. \& Pérez-Martínez, G. (1997). Establishing a model to study the regulation of the lactose operon in Lactobacillus casei. FEMS Microbiol Lett 148, 83-89.

Gosalbes, M. J., Monedero, V. \& Pérez-Martínez, G. (1999). Elements involved in catabolite repression and substrate induction of the lactose operon in Lactobacillus casei. J Bacteriol 181, 3928-3934.

Henstra, S. A., Duurkens, R. H. \& Robillard, G. T. (2000). Multiple phosphorylation events regulate the activity of the mannitol transcriptional regulator MtlR of the Bacillus stearothermophilus phosphoenolpyruvate-dependent mannitol phosphotransferase system. J Biol Chem 10, 7037-7044.

Ho, S. N., Hunt, H. D., Horton, R. M., Pullen, J. K. \& Pease, L. R. (1989). Site-directed mutagenesis by overlap extension using polymerase chain reaction. Gene 71, 51-59.

Houman, F., Diaz-Torres, M. R. \& Wright, A. (1990). Transcriptional antitermination in the $b g l$ operon of $E$. coli is modulated by a specific RNA binding protein. Cell 62, 1153-1163.

Idelson, M. \& Amster-Choder, O. (1998). SacY, a transcriptional antiterminator from Bacillus subtilis, is regulated by phosphorylation in vivo. J Bacteriol 180, 660-666.

Krüger, S. \& Hecker, M. (1995). Regulation of the putative $b g l P H$ operon for aryl- $\beta$-glucoside utilization in Bacillus subtilis. J Bacteriol 177, 5590-5597.

Krüger, S., Gertz, S. \& Hecker, M. (1996). Transcriptional analysis of $b g l P H$ expression in Bacillus subtilis: evidence for two distinct pathways mediating carbon catabolite repression. J Bacteriol 178, 2637-2644.

Le Coq, D., Lindner, C., Krüger, S., Steinmetz, M. \& Stülke, J. (1995). New $\beta$-glucoside $(b g l)$ genes in Bacillus subtilis: the bglP gene product has both transport and regulatory functions similar to those of BglF, its Escherichia coli homolog. J Bacteriol 177, $1527-1535$.

Leloup, L., Ehrlich, S. D., Zagorec, M. \& Morel-Deville, F. (1997). Single crossing-over integration in the Lactobacillus sake chromosome and insertional inactivation of the pts and the lacI genes. Appl Environ Microbiol 63, 2117-2123.

Lindner, C., Galinier, A., Hecker, M. \& Deutscher, J. (1999). Regulation of the activity of the Bacillus subtilis antiterminator LicT by multiple PEP-dependent, enzyme I- and HPr-catalysed phosphorylation. Mol Microbiol 31, 995-1006.

Mahadevan, S. \& Wright, A. (1987). A bacterial gene involved in transcription antitermination: regulation at a Rho-independent terminator in bgl operon of E. coli. Cell 50, 485-494. 
Mahadevan, S., Reynolds, A. E. \& Wright, A. (1987). Positive and negative regulation of $b g l$ operon in Escherichia coli. J Bacteriol 169, 2570-2578.

Marasco, R., Salatiello, I., de Felipe, M. \& Sacco, M. (2000). A physical and functional analysis of the newly-identified bglGPT operon of Lactobacillus plantarum. FEMS Microbiol Lett 186, 269-273.

Martin-Verstraete, I., Stülke, J., Klier, A. \& Rapoport, G. (1995). Two different mechanisms mediate catabolite repression of the Bacillus subtilis levanase operon. J Bacteriol 177, 6919-6927.

Martin-Verstraete, I., Charrier, V., Stülke, J., Galinier, A., Erni, B., Rapoport, G. \& Deutscher, J. (1998). Antagonistic effects of dual PTS-catalysed phosphorylation on the Bacillus subtilis transcriptional activator LevR. Mol Microbiol 28, 293-303.

Monedero, V., Gosalbes, M. J. \& Pérez-Martínez, G. (1997). Catabolite repression in Lactobacillus casei ATCC 393 is mediated by CcpA. J Bacteriol 179, 6657-6664.

Pérez-Martínez, G., Kok, J., Venema, G., van Dijl, J. M., Smith, H. \& Bron, S. (1992). Protein export elements from Lactococcus lactis. Mol Gen Genet 234, 401-411.

Porter, E. V. \& Chassy, B. M. (1988). Nucleotide sequence of the $\beta$ D-phospho-galactosidase gene of Lactobacillus casei: comparison to analogous $p b g$ genes of other Gram-positive organisms. Gene 62, 263-276.

Posno, M., Leer, R. J., van Luijk, N., van Giezen, M. J. F., Heuvelmans, P. T. H. M., Lokman, B. C. \& Pouwels, P. H. (1991). Incompatibility of Lactobacillus vectors with replicons derived from small cryptic Lactobacillus plasmids and segregational instability of the introduced vectors. Appl Environ Microbiol 57, 1822-1828.

Rutberg, B. (1997). Antitermination of transcription of catabolic operons. Mol Microbiol 23, 413-421.

Sambrook, J., Fritsch, E. F. \& Maniatis, T. (1989). Molecular Cloning: a Laboratory Manual, 2nd edn. Cold Spring Harbor, NY : Cold Spring Harbor Laboratory.

Schnetz, K. \& Rak, B. (1988). Regulation of $b g l$ operon of Escherichia coli by transcriptional antitermination. EMBO J 7, 3271-3277.

Schnetz, K. \& Rak, B. (1990). $\beta$-Glucoside permease represses the $b g l$ operon of E. coli by phosphorylation of the antiterminator protein and also interacts with glucose-specific enzyme II, the key element in catabolic control. Proc Natl Acad Sci USA 87, 5074-5078.

Schnetz, K., Toloczki, C. \& Rak, B. (1987). $\beta$-Glucoside (bgl) operon of Escherichia coli K-12: nucleotide sequence, genetic organization, and possible evolutionary relationship to regulatory components of two Bacillus subtilis genes. J Bacteriol 169, 2579-2590.

Schnetz, K., Stülke, J., Gertz, S., Krüger, S., Krieg, M., Hecker, M. \& Rak, B. (1996). LicT, a Bacillus subtilis transcriptional antiterminator protein of the BglG family. J Bacteriol 178, 1971-1979.

Stülke, J., Martin-Verstraete, I., Zagorec, M., Rose, M., Klier, A. \& Rapoport, G. (1997). Induction of the Bacillus subtilis ptsGHI operon by glucose is controlled by a novel antiterminator, GlcT. Mol Microbiol 25, 65-78.

Stülke, J., Arnaud, M., Rapoport, G. \& Martin-Verstraete, I. (1998). PRD - a protein domain involved in PTS-dependent induction and carbon catabolite repression of catabolic operons in bacteria. Mol Microbiol 28, 865-874.

Taranto, M. P. (1999). Reducción de colesterol e hidrólisis de sales biliares por bacterias lácticas. $\mathrm{PhD}$ thesis, Universidad Nacional de Tucumán, Argentina.

Tobisch, S., Stülke, J. \& Hecker, M. (1999). Regulation of the lic operon of Bacillus subtilis and characterization of potential phosphorylation sites of the LicR regulator protein by sitedirected mutagenesis. J Bacteriol 181, 4995-5003.

Tortosa, P., Aymerich, A., Lindner, C., Saier, M. H., Jr, Reizer, J. \& Le Coq, D. (1997). Multiple phosphorylation of SacY, a Bacillus subtilis antiterminator negatively controlled by the phosphotransferase system. J Biol Chem 272, 17230-17237.

Van Tilbeurgh, H., Le Coq, D. \& Declerck, N. (2001). Crystal structure of an activated form of the PTS regulation domain from the LicT transcriptional antiterminator. EMBO J 20, 3789-3799.

Viana, R., Monedero, V., Dossonnet, V., Vadeboncoeur, Ch., Pérez-Martínez, G. \& Deutscher, J. (2000). Enzyme I and HPr from Lactobacillus casei: their role in sugar transport, carbon catabolite repression and inducer exclusion. Mol Microbiol 36, 570-584.

Zukowski, M., Miller, L., Cosgwell, P., Chen, K., Aymerich, A. \& Steinmetz, M. (1990). Nucleotide sequence of the sacS locus of Bacillus subtilis reveals the presence of two regulatory genes. Gene 90, 153-155.

Received 10 July 2001; revised 15 October 2001; accepted 13 November 2001. 